\title{
Cerebellopontine Angle Schwannoma
}

National Cancer Institute

\section{Source}

National Cancer Institute. Cerebellopontine Angle Schwannoma. NCI Thesaurus. Code C5413.

A schwannoma occurring in the cerebellopontine angle. 